\title{
CLINICAL SURFACES - Activity-Based Computing for Distributed Multi-Display Environments in Hospitals
}

\author{
Jakob E. Bardram, Jonathan Bunde-Pedersen, Afsaneh Doryab, \\ and Steffen Sørensen \\ IT University of Copenhagen, Rued Langgaards Vej 7 \\ DK-2300 Copenhagen, Denmark \\ \{bardram, jonathan, adoryab, sts\}@itu.dk
}

\begin{abstract}
A multi-display environment (MDE) is made up of co-located and networked personal and public devices that form an integrated workspace enabling co-located group work. Traditionally, MDEs have, however, mainly been designed to support a single "smart room", and have had little sense of the tasks and activities that the MDE is being used for. This paper presents a novel approach to support activity-based computing in distributed MDEs, where displays are physically distributed across a large building. CLINICAL SURFACES was designed for clinical work in hospitals, and enables context-sensitive retrieval and browsing of patient data on public displays. We present the design and implementation of CLINICAL SURFACES, and report from an evaluation of the system at a large hospital. The evaluation shows that using distributed public displays to support activity-based computing inside a hospital is very useful for clinical work, and that the apparent contradiction between maintaining privacy of medical data in a public display environment can be mitigated by the use of CLINICAL SURFACES.
\end{abstract}

\section{Introduction}

A multiple display environment (MDE) is comprised of co-located personal and public devices, ranging from small devices (e.g. PDAs, laptops, and tablet PC) to large wall-based displays, which are networked to form an integrated workspace [4]. The goal of such MDEs is to foster co-located group work utilizing available devices.

An on-going research challenge is to design the technologies and user interfaces for such MDEs. Many systems and interaction techniques have been proposed [18, 8, $10,4,16,17]$, each addressing specific issues of creating an MDE. Existing research has, however, mainly focused on MDEs within a single physical space - often referred to as a Smart Space. Little work has addressed what we call a "Distributed Multi-Display Environment" (dMDE), i.e. an MDE that is distributed across several physical spaces inside e.g. a large building. Furthermore, most existing research has focused on information and device management for sharing basic data on the devices. For example, sharing and exchanging documents, spreadsheets, presentation, images, etc. Less work is available on supporting whole work tasks and activities. For example, how the different documents, data, and services involved in a specific work activity may belong together, and how an MDE may help collate and present these 
resources in a coherent and efficient manner for the people collaborating around this activity.

This paper proposes CLINICAL SURFACES, which is a set of user-interface technologies and infrastructure elements supporting a distributed multi-display environment with a special focus on supporting clinicians working inside hospitals. Hence, the type of dMDE we are designing for is a setup where displays are scattered around the entire hospital and are available in e.g. conference rooms, patient wards, hallways, and operating rooms.

The goal of CLINICAL SURFACES is to help clinicians use publicly available displays for managing large amount of medical data while moving between different physical spaces. Key features include support for easy access to an aggregation of all information and data relevant for a specific patient; access to this data from all displays in the dMDE; support for having several users actively participating in the work on a patient activity; support for moving patient activities and their related resources around in the dMDE; support for both fixed (wall-based) and mobile public displays; support for context-aware adaptation of the access to relevant activities; and support for a seamless transition between using the display in a public, personal, or private mode.

\section{Related Work}

Research on MDEs or Smart Spaces dates back to the original work on Ubiquitous Computing at PARC, which supported an integrated user experience ranging from small-size "pads" to medium-size "tabs" to large-size wall-based displays [19]. Similarly, the iLand [18], the Interactive Space [10] and the Gaia smart space projects [8] have been researching the infrastructure and interaction technologies for seamless integration of mobile and fixed displays in MDEs. Technologies like PointRight [11], ARIS [3], and IMPROMPTU [4] enable users to bind devices together, redirect control and user interfaces, and to share and move information across multiple displays. These projects all hold two things in common. One is that the technology is deployed inside one room only; the other is that besides from simple knowledge about who is in the room, the technology has no knowledge of the activity of the users. Our work deliberately focus on dMDEs as well as creating support for handling devices, information, services, and users in relation to the activities that is taking place in the dMDE.

Explicit computational support for human activity is the main focus for activitybased computing approaches. For example, activity-based computing support for the personal computer [2] illustrates how the computer can model the human activity and use this for information and window management in the Windows XP operating system. Similarly, the Unified Activity Management project [14] uses activities for information management and collaboration inside large organizations. More automatic systems based on sensor inference have demonstrated how activities can be inferred from low-level user interaction with the computer. For example, the context-aware activity display (CAAD) [15] is able to detect, cluster, and highlight activities that the user might be engaged in. This is used for semi-automatic information management of documents, files, web pages, and emails associated with an activity. 
None of these activity-based computing approaches address large interactive displays which can be used for public purposes in a dMDE, as CLINICAL SURFACES does. Furthermore, CLINICAL SURFACES is designed to highlight relevant activities in a specific contextual situation using context sensing of the physical world. This is different from the CAAD system, which is targeted towards activity recognition and highlighting based on low-level user input to a personal computer.

A particular set of semi-public display has been categorized as "awareness displays", i.e. displays that provide the user with peripheral information on the flow of work. Kimura [12] is an augmented office environment using interactive personal peripheral displays to manage "working contexts". The Notification Collage [7] is a system for users to communicate through a network of desk-mounted peripheral displays and public large displays. Because CLINICAL SuRfACES constantly lists and highlights relevant activities for its current context, it could work as an awareness display. The aim of CLINICAL SURFACES is, however, to support a more active engagement with the displays. Dynamo [9] enables collaboration around a large interactive display, but relies on the users to provide data and organize applications and content at each new session. Thus, Dynamo does not relieve the user of the overhead that also exists in regular PC usage. CLINICAL SURFACES similarly supports collaboration in a public setting, but in addition it explicitly supports the activity of a user and thereby decrease the work involved in starting applications, finding relevant data, etc.

\section{Background}

This work is based on extensive observations of clinical work in large hospitals, and on the design of pervasive computing technology for hospitals [1]. In many ways, the work of hospital clinicians is very different from that of information workers - the latter having been the primary target for the design of interactive computer technology. First of all, work in hospitals is nomadic, i.e. clinicians constantly move around inside the hospital visiting different patients, departments, wards, conference rooms, and colleagues. In contrast to mobile work, nomadic work is characterized by the fact that the clinicians do not have any place to sit and work, i.e. most clinicians do not have a desk or an office of any kind. Second, clinical work is intensively collaborative; patient care happens in close collaboration between a team of highly specialized clinicians who constantly need to align, coordinate, and articulate their respective part of the overall activity of treating the patient. This collaboration typically pivots around shared medical information available in medical records, medical charts, radiology images, etc. Moreover, collaboration is often co-located, i.e. clinicians meet for planned or ad-hoc conferences and discuss the patient case. Examples of such conference situations range from the formal medical conference every morning, to improvised and ad-hoc conferences between clinicians in the hallway of the ward.

Analyzing the work artifacts used in hospitals, one finds that publicly available "displays" play a core role in the execution and coordination of work. Examples include large whiteboards listing operations, patients, beds, and other critical resources; medical records; notebooks; and small notes. Some of these artifacts are fixed to the wall while others are mobile. However, common to them all is their public nature, that they are used as a shared collaborative resource. 


\section{CLINICAL SURFACES}

The design of CLINICAL SURFACES is based on the principles of (i) activity-based computing, use of (ii) fixed and mobile displays, (iii) context-based adaptation, and a separation between (iv) public, personal, and private use of these public displays.

Activity-based computing [2] includes support for activity centered computing, where resources and participants associated with a real-world human activity are modeled and aggregated in a corresponding computational activity. For example, the treatment of a patient may be modeled as a computational activity by aggregating all relevant medical information (e.g. medical records, radiology images, care plans, and medicine charts) as well as the participants involved in this activity. Each user participates in many activities, and the same activity may have several participants; the latter approach designed to support collaborative sharing of the resources associated with the activity. Moreover, activity-based computing includes support for activity roaming, i.e. support for moving a computational activity from one device to another; we say that an activity can be suspended on one machine and resumed on another. Activity-based computing is specifically targeted at supporting collaborative management of the myriad of heterogeneous data associated with a patient case, and to support the nomadic work inside e.g. hospitals.

The second principle is to support public displays. This is similar to the support for public wall-based displays in other MDEs, like iRoom or iLand. However, in addition to this previous work on supporting fixed wall-based displays in smart space technology, CliniCAL SuRfaCes is deliberately designed to also support mobile public displays. This resembles e.g. the use of the medical record in a hospital, which is a shared clinical artifact that is carried around by the clinicians.

Public displays constantly change usage context. Fixed public displays are frequently used by many different users for different activities, and mobile public displays are furthermore continuously changing their physical context. Hence, the design for public displays necessitates the support for these displays to adapt to their usage context. In CliniCAl SuRfaces this is achieved by monitoring the context of the display, and continuously highlighting and providing access to the most relevant activities in the current context.

Finally, even though CLINICAL SURFACES is primarily designed to support public displays, it is also designed to support a seamless transition to more personal and private use of it. Hence, a user may choose to appropriate any public display and transform it into a personal device.

\subsection{Interaction Design}

Figure 1 shows the user interface of CLINICAL SURFACES consisting of four main parts: (A) the context bar showing this public display's current context; (B) the activity list showing an overview of relevant activities within this context; (C) a resumed activity providing an overview of its associated resources; and (D) two applications launched as part of the resumed activity. This interface is used on all public displays and is able to scale from portable tablet PC to large wall-based displays. 

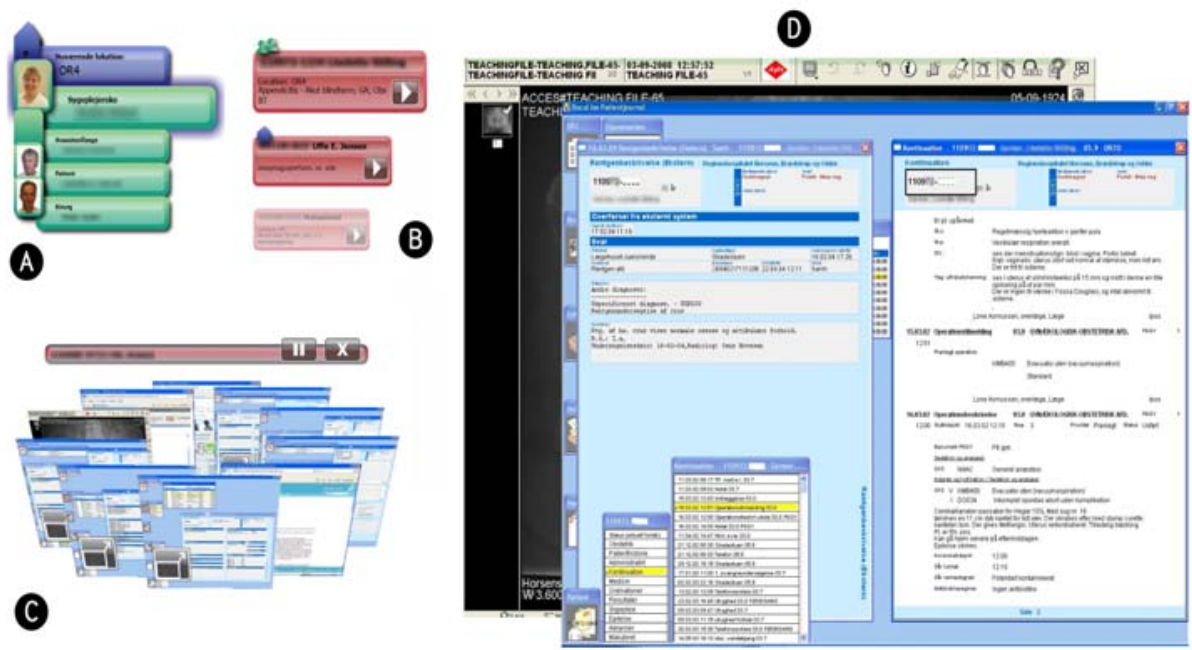

Fig. 1. The CLINICAL SURFACES user interface - (A) Context Bar (B) List of activities, (C) a resumed activity with associated resources displayed in a carrousel, and (D) two running applications

The context bar (A) shows the current context of this public display. This includes displaying the current location using a blue "house" icon, and nearby persons (including patients) using a green label and a picture. If the public display is fixed to e.g. a wall, the current location seldom changes. However, if this is a mobile public display, the current location is constantly tracked. Similarly, the display constantly tracks and displays a list of persons co-located with this public display.

The activity list (B) is constantly adjusted to show and highlight which activities are most relevant in this context. The current implementation operates with three levels of relevance shown as a large, small, and transparent red box. The icons attached to each activity reveal the type of relevancy: the icon with the two users illustrate that several persons co-located with the public display participate in this activity; the house icon illustrates that this activity is relevant for the current location of this public display.

An activity can be resumed by clicking the "Play" icon. Then CLINICAL SURFACES shows previews of its associated resources, as shown in Figure 1 (C). The user can now choose to either restore each resource individually by clicking the "Enlarge" icon on each preview, or s/he can restore the whole activity, i.e. all of the associated resources by clicking on the "Play" icon on the activity's title bar. Restoring a resource means that this resource is fetched and displayed using the local device's relevant application. Hence, a URL link opens in a web browser, a Word document in MS Word, etc. In Figure 1 (D), an electronic patient record and an X-ray image relevant to this patient are shown. The activity is suspended again by clicking the "Pause" icon, and the state of the activity is saved. This includes information about the placement and state of any resumed applications. Clicking on the "X" dismisses the "carousel" view. Each display can handle multiple resumed activities simultaneously, thereby enabling co-located use. 


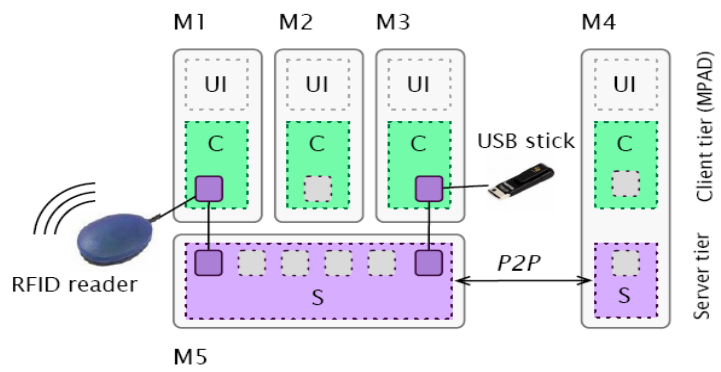

Fig. 2. The CLINICAL SURFACES architecture showing the (S)erver, (C)lient, and user interface (UI) components, as well as the extension points with e.g. RFID reader interface. This setup has five machines.

CliniCAL SURfaCES distinguishes between three levels of privacy; public, personal, and private. Public is the default mode where the relevancy algorithm puts equal weight to the information presence of all close-by personnel. The personal mode is entered by double-clicking on a person in the context bar. This is, for example, used when a clinician picks up a mobile public display. In the personal mode, the display shows all the activities that this person participates in, including the ones not relevant for the current context. Private mode is activated when a USB stick containing private activities is inserted into a display. Then context information is ignored, and the user can work only on the private activities stored or referenced on the USB stick. Once the USB stick is removed, the display reverts back to operate in a public mode.

The focus of CLINICAL SURFACES is to support easy access to activities and associated resources and participants. Hence, focus has not been on activity management, i.e. the creation and maintenance of activities. This is done in a semi-automatic fashion using context-aware technologies [2], which is beyond the scope of this paper.

\subsection{Technical Implementation}

CliniCAL Surfaces is implemented on top of a distributed storage and event system called AEXO. As illustrated in Figure 2, the system contains two tiers; a server tier and a client tier; the latter containing the user-interface (UI) is shown in Figure 1.

The server tier maintains a hierarchical map data structure holding all persistent data in the system, including users, activities, (links to) resources, and location and context information. AEXO embeds an event system that enables clients to listen for changes in the map. The server tier can be distributed in a peer-to-peer fashion across several host machines; like between M4 and M5 in Figure 2. AEXO dynamically listens for and mounts data, which may reside on external data sources such as laptops, USB drives, and RFID readers. This data is incorporated into an existing map. This feature is used to implement the feature of having private activities on a USB stick.

Communication between the AEXO client and server tiers can use several protocols, but the AEXO C\# client library layer wraps all aspects of the lower level communication details. The main responsibility of the AEXO client tier is to ensure that the CLINICAL SURFACES object model is synchronized with the hierarchical map in AEXO. This is done by adding $\mathrm{C \#}$ annotations of relevant properties in the data model. The user 
interface of CLINICAL SURFACES is implemented in Windows Presentation Foundation (WPF) using data binding to the underlying data model store in AEXO. This three-layer model ensures that events from e.g. changes in the AEXO data map are propagated to the AEXO clients, which updates the client object model, which again propagates changes to the CLINICAL SURFACES WPF user interface.

\section{Evaluation}

CliniCAL SuRfaCes has been evaluated in a large hospital. It was used during a full day ( 8 hours) by clinical personnel from the orthopedic department, including surgeons, operating nurses, anesthesiologists, and ward nurses. In total 8 clinicians participated. Since patients were not intended users, no real patients were involved; two staff members of the hospital helped playing the patient role. The acting patients, were, however treated as any real patient, including being physically examined by the doctors and prepared for surgery.

\subsection{Evaluation Setup}

The deployment of CLINICAL SURFACES in the hospital is illustrated in Figure 3. The locations include the conference room (A) used by the surgeons for the morning conference; the patient ward (B) where admitted patients are hospitalized; the hallway

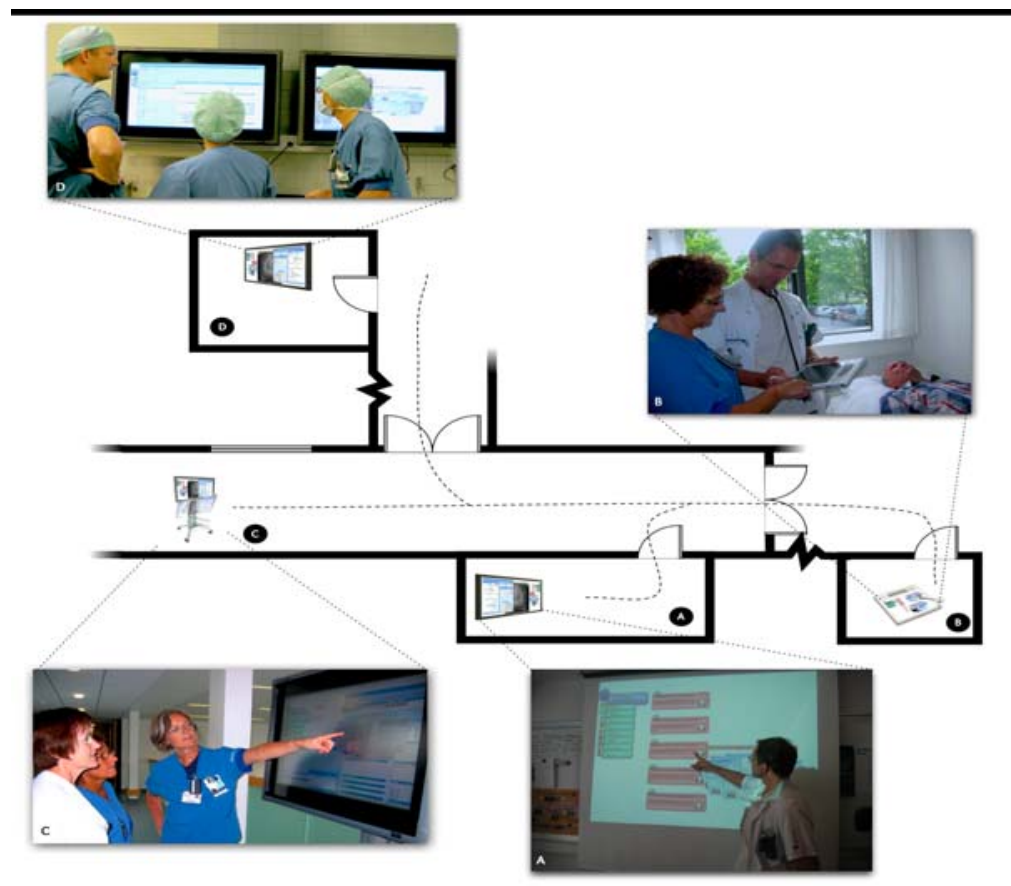

Fig. 3. The area of the hospital in which we evaluated the clinical prototype. (A) is the conference room, (B) the patient-ward, (C) the hallway, and (D) the operating room. 
leading to the patient ward (C); and inside an operating room (D). Wall-based public displays using 42" touch screens were deployed in the conference room, the hallway, and inside the OR. A Lenovo tablet PC and a Motion Computing C5 Clinical tablet PC were used as mobile public displays. The mobile displays were used mainly at the patient ward. All displays (both mobile and fixed) and all clinical personnel were tracked using active RFID tags (WaveTrend LRX400), which had a reading range of ca. 10 meters.

In total 15 real patient cases were modeled in CLINICAL SURFACES as activities. All of these activities had associated medical data from the electronic patient record, the radiology system, and various web-based medical applications. Figure 1 shows an example of the user interface of CLINICAL SURFACES with this data. The creation and maintenance of the activities was performed in a Wizard-of-Oz manner by a human operator [13]. As the focus was only browsing the relevant patient data and not the usage of the applications, screen shots of the real applications were used.

\subsection{Evaluation Methods}

The evaluation was not intended to be a strict usability evaluation but was adopted to test the design of CLINICAL SURFACES in a real clinical environment. The aim was thus to assess the feasibility of a new innovative design, in which case applying traditional usability evaluation in a lab setup would be rather inappropriate [6].

The evaluation was centered around a real patient case in an anonymized form. The entire patient case was authentic, including all medical data and events. The main flow of the case was:

1. The patient is admitted to the hospital for acute appendicitis during the night

2. In the morning, surgery is planned during the medical conference

3. The patient is visited at the bed ward

4. An ad-hoc discussion of the case takes place in the hallway

5. Surgery is prepared in the operation room

6. Surgery is performed

7. The patient is taken back to the bed ward

The goal was to provide objective measurements on the usefulness and usability of our design while, at the same time, investigating the underlying detailed user reaction to the system and the user interface in a more qualitative fashion. For this purpose, we used a multi-method evaluation setup where we (i) asked the users to enact the patient case as realistically as possible while thinking aloud, (ii) investigated perceived usefulness and usability based on a questionnaire [5], and (iii) made a semi-structured follow-up interview.

\section{Results}

The results from the perceived usefulness/ease of use questionnaire are shown in Table 1. Overall, the system is perceived very useful $(4.16$, std. dev. of 0.65$)$ and easy to use and learn (4.13, std. dev. 0.57). Looking at the specific aspects of the system, Table 1 also shows the perceived usefulness of the four main features of the system. 
Table 1. Perceived usefulness summary $(\mathrm{N}=8$, scale $=1-5)$

\begin{tabular}{lll} 
Perceived usefulness & Avg. & Std. dev. \\
\hline System usefulness & 4.16 & 0.65 \\
Ease of using and learning & 4.13 & 0.57 \\
Activity Management & 4.25 & 0.52 \\
Public Displays & 4.34 & 0.69 \\
Context-based adaptation & 3.56 & 0.84 \\
Public-Personal-Private & 4.22 & 0.79 \\
\hline
\end{tabular}

\subsection{Activity Management}

Support for activity management scores high - 4.25 (std. dev 0.52). Hence, the clinicians either agreed or strongly agreed that it would be useful to gather all relevant patient information in an activity, that an activity would help recover relevant patient data quickly, and that it would be easy to get access to patient data while moving around inside the hospital. As argued by a surgical nurse in the subsequent interview:

Such a system will definitely be a huge advantage. Everything would be collated in one place. Today, we spend a lot of time in getting access to the different systems, and to find the right documentation in them.

Similarly, the simple overview that CLINICAL SURFACES provides was highly appreciated by the clinicians as an important feature in a busy work environment:

What I like about the system is that we all use different aspect of the clinical data, and I can just select the parts that I need. I think the user interface is extremely simple.

During the discussion, the clinicians also suggested things for improvement. For example, having mechanisms for displaying activities and services in fixed locations on the screen might increase the overview and help the clinicians to quickly locate important information.

\subsection{Public Displays}

Using public displays in a clinical environment was considered very useful (4.34, std. dev 0.69). Hence, the clinicians agreed that fixed and mobile displays would be a good fit to the working conditions of a hospital; that it would be important to have the same interface on both the wall-based and the mobile displays; and that being able to just walk up to a public display and start using it would be a crucial feature. As expressed by a nurse:

Another good thing about this system is, that in a busy hospital [...] you do not need to spend time accessing data. You just go to the screen and get the data. [...] One can imagine that there are 10 of these mobile displays for each [patient] ward and you just take one from a re-charger station when you enter the ward.

The participants also discussed where to place wall-based and mobile displays. They concluded that the smaller mobile displays would suit the work in a patient 
ward, whereas it would be of little use inside the operating room. In the operating room, the large wall-based displays would be useful since they display more data and can be viewed from a distance. Furthermore, carrying mobile display in and out of operating room also implies a contamination hazard.

\subsection{Context-Based Adaptation}

The feature of context-based adaptation scores consistently low (3.56, std. dev 0.84). In particular, the clinicians did not agree that CLINICAL SURFACES was displaying the most relevant activities for a specific situation, and that using location tracking of personnel would help find the most relevant activities. Rather than co-location of people in front of the display, it seemed that the relevance of activities - or more specifically the patient cases - depended more on clinical issues like urgency of the case, or the order of the operations to be done in the operating room. As argued by a surgeon:

On the operation hallway - if we want to have information about a patient then we can go to the display and activate the name. But in the operation room, it is perhaps better to have the patients' [activities] fixed on the screen so that they will not change or move.

Another issue with the adaptation is that the content and appearance of the activity list would change based on people entering and leaving the room - as expressed by an operation nurse:

When, for example, [the surgeon] enters the operation room he can see his own activities and can see what he is going to do after this process. And it is really good for [him]. But the team in the operating room may be confused if his presence in the room starts to affect the tasks, which are linked to the people in this room.

\subsection{Public-Personal-Private}

Smooth transition between a public, personal, and private use of the displays also scores high on perceived usefulness (4.22, std. dev 0.65). The clinicians clearly agreed that it would be beneficial to see both shared as well as personal activities, and they also argued that in most cases, privacy is less of a problem, since ownership of patient related clinical data needs to be shared among all parties involved in the treating of a patient. As argued by a ward nurse:

...it is good that only you have access to the private data on the USB [stick] and not others, but as long as it is patient information and something which everybody has an interest in, it is nice to be able to transfer the screen to another [member of the staff].

The clinicians, however, pointed out that it was important to consider exactly where to put the public wall-based displays; they should be located in places where people, irrelevant for the patient case (e.g. other patients and relatives), would not accidentally view the data or overhear a conversation. In the evaluation setup, the display in the hallway was placed in a space too crowded and too public. 


\section{Discussion}

In this section, we want to discuss lessons from the evaluation of CLINICAL SURFACES in relation to existing and future research.

\subsection{Activity-Based dMDEs}

CliniCAL SuRfaCES supports bundling of related services and data which is associated with some real-world human activity, like the patient cases used in the evaluation. The clinicians agreed that this support for activity-centered bundling of resources is very useful. Furthermore, the support for roaming the activities between public displays, as enabled by the AEXO platform, was deemed essential for work inside the hospital.

The distributed nature of our interactive space is very unlike most related research on MDEs. The physical disconnectedness of the devices and displays in a dMDE is a crucial point of difference. The structure of hospital work and the architecture of hospitals themselves do not easily lend themselves to the application of MDE as a technology confined in isolated rooms. Instead hospitals have vast corridors, offices, operating rooms and patient wards. The approach we have taken is to consider the entire hospital as an interactive space and deploy multiple displays in such an environment.

This has raised a number of new research challenges, which traditional MDEs do not address. Among these, we found two issues to be of central importance for a dMDE; how to enable the flow of activities and data between the public displays, and how to enable users to access and start using the displays in the dMDE. In CLINICAL SURFACES, the first challenge is addressed by using location information to automatically direct relevant activities onto a wall-based public display. Hence, activities and their associated resources - were simply just following the users around inside the dMDE. Based on the feedback from the clinicians, this approach seemed to work well.

Furthermore, by using mobile devices, activities could be "carried" wherever they were needed, and this fact was used in the case of the ward-rounds. In contrast to traditional use of tablet PCs, CLINICAL SURFACES also turned this device into a public display participating in the dMDE. In several cases, the tablet PCs were handed over from one clinician to another (as shown in Figure 3B). In these 'hand-over' situations, the display simply switched context and was carried for use elsewhere in the dMDE.

Finally, to support accessing and carrying private activities - with associated data the USB stick served as a "pocket-book"; i.e. a private store for activities which could be inserted in all fixed and mobile screens thereby converting them to personal devices. This kind of local adaptation of specific displays in the dMDE is another important difference from a traditional MDE.

\subsection{Public Displays and Privacy}

There seems to be an inherent conflict between using public displays and maintaining privacy. The latter is especially important in a clinical domain where sensitive patient data is displayed and discussed. As outlined in previous section, this issue was also 
discussed by the clinicians while using CLINICAL SURFACES in the hallway. It was a deliberate choice to put the display in this rather public location, hoping to spark a discussion of the feasibility of having such public displays around the hospital. The discussion, however, was concerned with several aspects of privacy. The first aspect was whether it is appropriate that one participant can see and access the activities of another participant present in front of the display. For example, in Figure 1 the surgeon has access to the nurse's activities. After some discussion, the clinicians concluded that this was actually not a privacy issue. Partly because all patients hospitalized at the hospital in principle should be accessible by all clinicians, and partly because the activities were only accessible if the user was in physical proximity of the display. Once the user moves away, his or her activities are no longer present in the display. Even though the reading range of the active RFID tags seemed to be too long in the deployment setup, the clinicians agreed that this design was suitable for upholding of privacy in hospital-specific activities. By using the USB stick - either as a key or as a holder of data - the users were able to more precisely control when private activities were displayed.

Another aspect of privacy was concerned with the physical deployment of such large wall-based displays. The deployment location of the hallway display was not appropriate in the opinion of the users; it was simply put in a location where too many people were passing by, including relatives and other patients. Hence, it would not be feasible to discuss a patient case in this location. The clinicians concluded that such displays would be very useful if put in the hallway of the operation ward or the patient ward. Inside the operation room, there were no privacy issues.

In summary, the inherent conflict between using public displays and the concern for privacy can be mitigated through the proper modeling of public, personal, and private activities in CLINICAL SURFACES and its infrastructure, combined with an appropriate deployment of the displays and tracking technology.

\subsection{Context-Based Relevance}

In the current implementation, context-based adaptation is linked to the physical copresence of participants of an activity; the more participants present in the same location as the public display, the more relevant the activity becomes. For the clinicians, this model was too simple - physical co-location could be a relevance-indicator, but a series of other more clinically oriented issues seems to be more important to incorporate. For example, the operation schedule of the day, the urgency of the patient case, and whether the case needed to be discussed on the morning conference. The current relevance score based on co-location also had an annoying side-effect, because when an RFID tag became invisible due to other reasons than the person leaving the room (e.g. being covered by something), then the relevance of the activities would immediately change, which would resize and re-organize the list of activities. This caused a lot of confusion during the evaluation. Since the technical implementation of CLINICAL SURFACES allows for replacement of the relevance algorithm, a natural next step would be to investigate how to improve this based on e.g. more clinical context information. 


\section{Conclusion}

In this paper, we have presented a novel approach and user interface technology for supporting activity-based computing in distributed multi-display environments named CLINICAL SURFACES. Taking an outset in the nomadic and collaborative work inside hospitals, CLINICAL SURFACES was designed to enable easy access to medical data across physically distributed public displays. CLINICAL SURFACES was evaluated in a large hospital and the evaluation revealed that the clinicians found the system very useful and easy to use. The support for activity management, distribution of clinical data, and the ability to quickly access all relevant data on any public display were considered as strong features. The evaluation, however, also pointed to areas for further research and improvements, including improving the current implementation of context-based adaptation.

\section{References}

1. Bardram, J.E., Christensen, H.B.: Pervasive Computing Support for Hospitals: An Overview of the Activity-Based Computing Project. In: IEEE Pervasive Computing, pp. 44-51 (2007)

2. Bardram, J.E., Bunde-Pedersen, J., Soegaard, M.: Support for activity-based computing in a personal computing operating system. In: Proceedings of CHI 2006, pp. 211-220 (2006)

3. Biehl, J.T., Bailey, B.P.: Aris: An interface for application relocation in an interactive space. In: Proceedings of Graphics Interface 2004, pp. 107-116 (2004)

4. Biehl, J.T., et al.: Impromptu: a new interaction framework for supporting collaboration in multiple display environments and its field evaluation for co-located software development. In: Proceedings of CHI 2008, pp. 939-948 (2008)

5. Davis: Perceived usefulness, perceived ease of use, and user acceptance of information technology. MIS Quarterly 13(3), 318-340 (1989)

6. Greenberg, S., Buxton, B.: Usability evaluation considered harmful (some of the time). In: Proceeding of CHI 2008, New York, NY, USA, pp. 111-120 (2008)

7. Greenberg, S., Rounding, M.: The notification collage: posting information to public and personal displays. In: Proceedings of CHI 2001, USA, pp. 514-521 (2001)

8. Hess, C., Romn, M., Campbell, R.: Building Applications for Ubiquitous Computing Environments. In: Proceedings of Pervasive 2002, pp. 16-29. Springer, Heidelberg (2002)

9. Izadi, S., Brignull, H., Rodden, T., Rogers, Y., Underwood, M.: Dynamo: a public interactive surface supporting the cooperative sharing and exchange of media. In: Proceedings of UIST 2003, pp. 159-168 (2003)

10. Johanson, B., Fox, A., Winograd, T.: The interactive workspaces project: Experiences with ubiquitous computing rooms. IEEE Pervasive Computing 1(2), 67-74 (2002)

11. Johanson, B., Hutchins, G., Winograd, T., Stone, M.: PointRight: experience with flexible input redirection in interactive workspaces. In: Proceedings of UIST 2002, pp. 227-234 (2002)

12. MacIntyre, B., Mynatt, E.D., Vodia, S., Hansen, K.M., Tullio, J., Corso, G.M.: Support for Multitasking and Background Awareness Using Interactive Peripheral Displays. In: Proceeding of UIST 2001, pp. 11-14 (2001)

13. Molin, L.: Wizard-of-oz prototyping for co-operative interaction design of graphical user interfaces. In: Proceedings of NordiCHI 2004, pp. 425-428 (2004) 
14. Moody, P., et al.: Business activity patterns: A new model for collaborative business applications. IBM Systems Journal 45(4), 683-694 (2006)

15. Rattenbury, T., Canny, J.: CAAD: An Automatic Task Support System. In: Proceedings of CHI 2007, pp. 687-696 (2007)

16. Rekimoto, J., Saitoh, M.: Augmented surfaces: a spatially continuous work space for hybrid computing environments. In: Proceedings of CHI 1999, pp. 378-385 (1999)

17. Stefik, M., et al.: Beyond the chalkboard: computer support for collaboration and problem solving in meetings. Commun. ACM 30(1), 32-47 (1987)

18. Streitz, N.A., et al.: i-land: an interactive landscape for creativity and innovation. In: Proceedings of CHI 1999, pp. 120-127 (1999)

19. Weiser, M.: The Computer for the 21st Century. Scientific American 265(3), 66-75 (1991) 